\title{
The influence of self-construals on the ERP response to the rewards for self and mother
}

\author{
Xiangru Zhu ${ }^{1,2} \cdot$ Huijun Zhang ${ }^{3} \cdot$ Lili Wu $^{4,5} \cdot$ Suyong Yang ${ }^{6} \cdot$ Haiyan $\mathrm{Wu}^{5,7} \cdot$ Wenbo Luo $^{8} \cdot$ Ruolei Gu $^{5,7} \cdot$ Yue-jia Luo $^{9}$
}

Published online: 20 February 2018

(C) Psychonomic Society, Inc. 2018

\begin{abstract}
Individual self-construal (independent vs. interdependent) could be temporarily modulated by the priming effect. Our previous studies have found that when Chinese participants gambled for mother and for self, outcome feedback evoked comparable neural responses between two conditions. However, it remains unclear if the response to rewards for mother and for self would differ after independence self-construal priming. In this study, we manipulated participants' selfconstrual (independent vs. interdependent) before a simple gambling task. The event-related potential (ERP) results reveal that when an interdependent self-construal was primed, the participants exhibited a comparable feedbackrelated negativity (FRN) elicited by outcome feedback for self and for mother. In contrast, independent self-construal priming resulted in a greater FRN elicited by outcome feedback for self than for mother. Meanwhile, the P3 component was insensitive to self-construal manipulation. These findings indicate the modulation effect of self-construal priming on the response to rewards for others.
\end{abstract}

Keywords Self-construals $\cdot$ Self $\cdot$ Mother $\cdot$ Outcome evaluation $\cdot$ Feedback-related negativity

Ruolei Gu

gurl@psych.ac.cn

1 Institute of Cognition, Brain and Health, Henan University, Kaifeng 475004, China

2 Institute of Psychology and Behavior, Henan University, Kaifeng 475004, China

3 School of Management, Guangdong University of Technology, Guangzhou 510520, China

4 CAS Key Laboratory of Mental Health, Institute of Psychology, Beijing 100101, China

5 Department of Psychology, University of Chinese Academy of Sciences, Beijing 100049, China

6 Department of Psychology, Shanghai University of Sport, Shanghai 200438, China

7 CAS Key Laboratory of Behavioral Science, Institute of Psychology, Beijing 100101, China

8 Liaoning Normal University, School of Psychology, Dalian 116029, China

9 Institute of Affective and Social Neuroscience, Shenzhen University, Shenzhen 518060, China

\section{Introduction}

Vicarious reward means that observing someone else receiving reward is rewarding in itself (Mobbs et al., 2009). The ability to vicariously experience the reward for others is critical in prosocial behaviors, but that experience is not as strong as the experience of the reward for oneself (Lockwood, Apps, Valton, Viding, \& Roiser, 2016). However, the mother in Chinese culture is a notable exception, as two of our eventrelated potential (ERP) studies have found that when Chinese participants gambled for mother and for self, outcome feedback evoked a comparable ERP response between two conditions (X. Zhu, Wang, et al., 2016; X. Zhu, Zhang, et al., 2015). This finding is consistent with the fact that in East Asian cultures, the neural representation of the self and that of mother largely overlap. For instance, the medial prefrontal cortex (mPFC) is an important brain region involved in selfprocessing (Macrae, Moran, Heatherton, Banfield, \& Kelley, 2004). Previous studies have observed comparable activations in the MPFC between making judgments about self and about mother in Chinese participants (G. Wang et al., 2012; Wuyun et al., 2014; Y. Zhu, Zhang, Fan, \& Han, 2007), but not in Western participants (Heatherton et al., 2006; Ray et al., 
2010). In our opinion, the undifferentiated neural representation between self and mother may contribute to the comparable ERP response to reward for mother and for self in Chinese culture.

Meanwhile, self-construal can modulate the response to rewards for self versus for others. Self-construal refers to how individuals define and make meaning of the self (Cross, Hardin, \& Gercek-Swing, 2011; Han \& Humphreys, 2016). Markus and Kitayama (1991) proposed two types of selfconstruals. Specifically, the independent self is a selfcontained and autonomous entity that is context-independent and includes salient internal attributes; in contrast, the interdependent self is treated as a member of a group, highlighting the belonging to and dependence on a context. Both independent and interdependent self-construal can coexist inside the same individual (Gardner, Gabriel, \& Lee, 1999). Remarkably, individuals from both individualistic and collectivistic nations have shown similar effects of self-construal on self-concept (Gardner et al., 1999). The self-construal type that is salient at one specific moment can modulate cognitive processing at both behavioral and neural levels (Colzato, de Bruijn, \& Hommel, 2012; Obhi, Hogeveen, \& Pascual-Leone, 2011; Sui, Hong, Hong Liu, Humphreys, \& Han, 2013; Van Baaren, Maddux, Chartrand, De Bouter, \& van Knippenberg, 2003).

In a recent functional magnetic resonance imaging (fMRI) study, Varnum, Shi, Chen, Qiu, and Han (2013) have found that participants were more sensitive to wins when gambling for self than for a friend after independent self-construal priming; in contrast, when participants were primed with interdependent self-construal, winning money for themselves and for a friend evoked comparable activations in the ventral striatum, which is an important brain region for reward processing. However, the flexibility of the influence of self-construal on the response to rewards for self versus for others needs further investigations. Although people experience rewards for a friend as strongly as for themselves under the interdependence priming, it remains unclear whether transient independent self-construal priming can differentiate the neural response to rewards for mother versus for self in Chinese culture.

We predicted that independent self-construal would make Chinese individuals to be more sensitive to their own reward than to mother's reward. This prediction is supported by the following facts: first, compared with interdependent selfconstrual priming, independent self-construal priming can enhance the level of self-awareness, making individual self to be more salient. For example, neural activity in the right medial frontal cortex was stronger when participants made judgments about their own face compared to another familiar face, and this difference was larger when participants were primed with independent self-construal compared to interdependent selfconstrual (Sui \& Han, 2007). Second, individuals are more sensitive to their reward when their uniqueness is emphasized.
One of our recent studies reported that outcome feedback in a gambling task evoked a larger difference wave of the feedback-related negativity (dFRN; see below) in the independent self-construal condition than in the interdependent selfconstrual condition (X. Zhu, Wu, Yang, \& Gu, 2017). Third, self-construal can also modulate the difference in brain activation between the "mother" and "self" conditions. For instance, Ng, Han, Mao, and Lai (2010) have found that the priming of Chinese culture decreased $\mathrm{mPFC}$ activations that differentiated between trait judgments about self and about mother, whereas the priming of Western culture produced the opposite effect.

For the purpose of the current study, the ERP technique was applied, which is well suited to investigate the dynamics of cognitive and social processes regarding its exquisite temporal resolution (Amodio, Bartholow, \& Ito, 2014). The feedback-related negativity (FRN) is one of the major ERP components associated with the outcome evaluation process (Gehring \& Willoughby, 2002; Miltner, Braun, \& Coles, 1997). The FRN is a medial frontal negative-flowing deflection that peaks approximately $250 \mathrm{~ms}$ following outcome feedback, being larger following negative compared to positive performance feedback (Miltner et al., 1997) and also larger following monetary losses compared to wins (Gehring \& Willoughby, 2002). This component may represent a cognitive system evaluating outcomes along a good-no good continuum (Foti, Weinberg, Bernat, \& Proudfit, 2015), which therefore makes it a useful tool for probing individual reward sensitivity (Bress \& Hajcak, 2013; Lange, Leue, \& Beauducel, 2012). A large body of ERP research has linked the FRN with the processing of reward prediction error (for a review, see Sambrook \& Goslin, 2015). Localization studies suggest that the FRN is generated in the mPFC (M. X. Cohen, Wilmes, \& van de Vijver, 2011; Walsh \& Anderson, 2012), which is consistent with fMRI findings about the role of $\mathrm{mPFC}$ in performance monitoring (e.g., Nieuwenhuis et al., 2005).

The P3 component is another ERP component related to outcome evaluation (San Martín, 2012; Yeung \& Sanfey, 2004). This component is sensitive to the emotional significance of an ongoing event; heightened P3 amplitudes indicate stronger emotional impact of an event (Hajcak, Dunning, \& Foti, 2009; Polezzi, Sartori, Rumiati, Vidotto, \& Daum, 2010). With regard to outcome evaluation, the $\mathrm{P} 3$ component is sensitive to reward magnitude and outcome uncertainty in monetary gambling tasks (San Martín, 2012). Previous studies that explored the ways in which self-construal affects the ERPs suggest that temporal self-construal priming modulates the early automatic components (e.g., the N1 component) but does not affect the late controlled components in the $\mathrm{P} 3$ time window (Jiang, Varnum, Hou, \& Han, 2014; C. Wang, Ma, \& Han, 2014). More relevant to the present study, two of our previous ERP studies have found that the P3 amplitude is insensitive to the contrast between self-reflection and other- 
reflection, indicating that the self-reflection priming effect does not occurred at the late stage of outcome evaluation (X. Zhu, Gu, Wu, \& Luo, 2015a; X. Zhu et al., 2017).

We hypothesized that the neural responses to rewards for mother and for self can be modulated by self-construal manipulation. Specifically, the neural responses to rewards for mother and self would be similar in the interdependent selfconstrual condition, but these responses would be weaker for mother than for self in the independent self-construal condition. In the present study, participants received independent and interdependent priming in different task blocks. After the priming stage, they performed a gambling task in which they were asked to gamble for themselves or their mothers. We predicted that interdependent self-construal priming would lead to similar FRN responses to rewards for self and mother, whereas independent self-construal priming would lead to stronger FRN responses to rewards for self than for mother. In addition, we predicted that the $\mathrm{P} 3$ component would not be modulated by self-construal priming.

\section{Methods}

\section{Participants}

A priori power analysis with $\mathrm{G}^{*}$ Power (version 3.1.7; Faul, Erdfelder, Lang, \& Buchner, 2007) revealed that a total sample size of 24 participants would provide about $82 \%$ statistical power for small-to-medium effect sizes. According to the suggestion from Vazire (2016), researchers should ensure that the power value does not fall short of $80 \%$.

Twenty-four college students (mean age: $21.6 \pm 0.6$ years, age range: $20-23 ; 14$ males) participated in this study. All participants were from Henan province, which is located in inner China. The experiment was approved by the Ethics Committee of the Department of Psychology in Henan University and was conducted in accordance with the 1964 Declaration of Helsinki. Informed consent was obtained prior to the experiment. All had normal vision (with correction), and none had a history of neurological disease or brain injury. All participants were right-handed.

\section{Procedure}

This study employed the priming of temporary self-construal, which has been widely used in previous research (e.g., Chiao et al., 2010; Krishna, Zhou, \& Zhang, 2008; Obhi et al., 2011; van Prooijen \& Zwenk, 2009). Also, the validity of this method has been confirmed in Chinese participants (e.g., Sui \& Han, 2007; Sui et al., 2013; Varnum et al., 2013; C. Wang et al., 2014).

There were two within-subject factors in the experiment, that is, Self-construal Type (two levels: independent/ interdependent self-construal) and Beneficiary (two levels: gamble for self/for mother). This $2 \times 2$ task design resulted in four separate blocks, the sequences of which were counterbalanced across participants. The use of a withinsubjects design is common in self-construal research field (Sui \& Han, 2007; Sui et al., 2013; Varnum et al., 2013).

Before the beginning of each block, one Chinese essay was presented for the self-construal priming procedure. The contents of the essays in four blocks were not the same, but they all consisted of two paragraphs describing a trip to the countryside (about 300-350 words) (Sui \& Han, 2007). The independent self-construal essays contained independent pronouns (e.g., I, mine), and the interdependent self-construal essays contained interdependent pronouns (e.g., we, ours). Participants were required to read each essay and to circle specific pronouns. All the essays used in the task were adopted from Sui and Han (2007).

Participants were told that the reading of essays was not related to the follow-up task, which was a forced-choice gambling game (Gu, Wu, Jiang, \& Luo, 2011). Before the experiment, participants were instructed about the rules and the meanings of the symbols in the gambling task. They were encouraged to respond in a way that would maximize the total score amount. The higher the score they earned, the more bonus money they or their mothers would receive after the experiment.

During the task, participants sat comfortably in an electrically shielded room approximately $80 \mathrm{~cm}$ from a computer screen. Stimulus display and behavioral data acquisition were conducted using E-Prime software (Version 1.1, Psychology Software Tools, Inc.). After the self-construal priming (see above), a beneficiary cue ("gamble for self/for mother") was presented $(3,000 \mathrm{~ms})$ at the beginning of each gambling block. Each trial began with the presentation of two white rectangles $\left(2.5^{\circ} \times 2.5^{\circ}\right.$ of visual angle) in which an Arabic number ("5") was individually presented to indicate two alternative options on the left and right sides of a fixation point. The number " 5 " represents 0.5 Chinese RMB Yuan. Participants were asked to make a selection by pressing the " $F$ " or " $J$ " key on the keyboard with the left or right index finger, respectively. The alternatives remained on the screen until the participants chose a rectangle, which was then highlighted by a thick red outline for $500 \mathrm{~ms}$. Thereafter, the outcome feedback of the participants' choice was presented such that its valence was displayed, with a 800- to 1,200-ms interval between them (see Fig. 1). Each block consisted of 96 trials. At the end of each block, participants completed a 7-point Likert scale (1: very unhappy; 7: very happy) to indicate how happy they felt when the beneficiary (self/mother) of the past block won, and another 7-point scale to indicate how unhappy they felt when the beneficiary lost. In total, each participant completed eight scales to measure happiness associated with outcome feedback in each condition. 


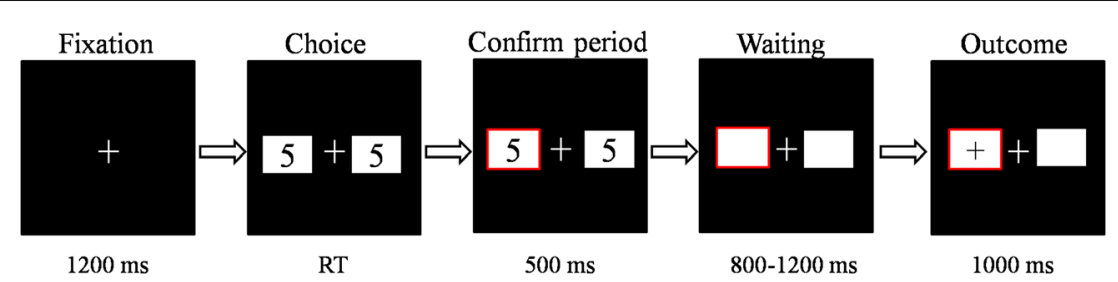

Fig. 1 The sequences of events within a single trial of the monetary gambling task. $R T$ response time

There were two kinds of outcomes: " +5 " and " -5 ," indicating the points the participants won and lost in the current trial, respectively. Unbeknown to the participants, the outcome feedback was provided according to a pre-determined pseudorandom sequence, such that each participant received exactly 48 of each kind of outcome regardless of their selection (left/right).

After participants finished the task, they were briefed that there was no optimal strategy for the task. Each participant and his/her mother were both paid 40 Chinese RMB Yuan (approximately US\$6) for remuneration. Regarding the reward for mothers, participants were asked to provide their mothers' cellphone number, and then the experimenter refilled 40 Yuan to that number via online service. The reliability of this method has been proved in our previous studies (e.g., X. Zhu, Wang, et al., 2016; X. Zhu, Wu, Yang, \& Gu, 2016b). Also, participants completed the Self-Construal scale, which is a 7-point Likert scale consisted of 24 items (Singelis, 1994). Internal consistency and test-retest reliability of the Chinese version of this scale were 0.88 and 0.78 , respectively (Y. Wang, Yuan, \& Xu, 2008). This scale has been successfully used to measure the levels of independent and interdependent self-construals of Chinese college students (Varnum et al., 2013).

\section{Electrophysiological recording and measures}

Electroencephalogram (EEG) activity was recorded from 63 scalp sites using tin electrodes mounted in an elastic cap (Brain Products) with an online reference to the middle at $\mathrm{FCz}$, and the data were off-line re-referenced to the global average reference. Electrode $\mathrm{FCz}$ was re-instated (Zendel \& Alain, 2014). Horizontal electro-oculogram (HEOG) was recorded from an electrode placed at the outer canthi of the right eye. Vertical electro-oculogram (VEOG) was recorded from an electrode placed above the left eye. All inter-electrode impedance was maintained at $<10 \mathrm{k} \Omega$. EEG and EOG signals were amplified with a bandpass from $0.05 \mathrm{~Hz}$ to $100 \mathrm{~Hz}$ and continuously sampled at $500 \mathrm{~Hz} /$ channel.

Off-line analysis of the EEG was performed using Brain Vision Analyzer software (Brain Products). The first step in data preprocessing was the correction of ocular artifacts using Independent Component Analysis (ICA) of the continuous data. The ocular-artifact-free EEG data were low-pass filtered below $30 \mathrm{~Hz}$ (48 dB/oct) and high-pass filtered above $0.05 \mathrm{~Hz}$
(48 dB/oct). Separate EEG epochs of 1,000 ms (including a baseline of $200 \mathrm{~ms}$ ) were extracted offline for the stimuli. All trials in which EEG voltages exceeded a threshold of $\pm 75 \mu \mathrm{V}$ during the recording epoch were excluded from data analysis. On average, about three trials per condition were excluded for each participant.

According to previous studies, the FRN amplitude can be calculated in essentially two ways: using grand-averaged waveforms or creating a difference wave between error and correct trials (Holroyd, Pakzad-Vaezi, \& Krigolson, 2008; Leng \& Zhou, 2010; Wu \& Zhou, 2009). The current study employed the difference-wave approach for two reasons. First, the main advantage of this method is the minimization of potential overlaps between the FRN and other ERP components, particularly the $\mathrm{P} 3$ (for detailed discussions, see Hajcak, Moser, Holroyd, \& Simons, 2007; Holroyd \& Krigolson, 2007). Indeed, the application of the difference wave method generates a clear FRN (Proudfit, 2015). Second, our previous studies have consistently used difference waves to measure the FRN (X. Zhu, Gu, et al., 2015; X. Zhu, Wang, et al., 2016; X. Zhu, Wu, et al., 2016; X. Zhu et al., 2017). Therefore, applying the same method would facilitate comparisons between the current data and our previous findings. Accordingly, we created the dFRN by subtracting the mean ERP amplitude in gain trials from that in loss trials within the 220- to 320-ms time window (Gu, Huang, \& Luo, 2010b; X. Zhu, Gu, et al., 2015). The electrode at which the dFRN reached their maximum was detected in the frontocentral area (FC1, FCz, FC2, C1, Cz, and C2). Arithmetical means were averaged across the six sites for data analyses. The dFRN amplitude analysis included two within-subjects factors, that is, Self-construal Type (independent/interdependent self-construal) and Beneficiary (self/mother).

We also evaluated the P3 (350-550 ms) amplitude by identifying its mean amplitude at the Pz electrode position where this ERP component typically reaches its maximum (e.g., Gu et al., 2017; Gu, Ge, Jiang, \& Luo, 2010a). The P3 amplitude analysis included three within-subject factors, that is, Outcome (win/loss), Self-construal Type (independent/interdependent self-construal), and Beneficiary (self/mother).

Statistical analysis was performed using IBM SPSS 19.0 (IBM Corporation). For all the analyses, the results of descriptive statistics were reported as mean \pm standard deviation (SD). The significance level was set at $p=.05$. The $p$ values were corrected for deviations according to Greenhouse and 
Geisser (1959). Partial eta-squared $\left(\eta_{p}^{2}\right)$ was reported to demonstrate the effect size of the variance of analysis (ANOVA), where 0.05 represents a small effect, 0.1 represents a medium effect, and 0.2 represents a large effect (J. Cohen, 1973).

\section{Results}

\section{Behavioral results}

Participants were highly accurate (more than $90 \%$ ) regarding their pronoun judgments in both the independent and interdependent self-construal conditions; therefore we did not further analyze the accuracy rate across conditions.

A $2 \times 2$ repeated-measures ANOVA involving Selfconstrual Type and Beneficiary was conducted on the reaction times (RTs) during the gambling task $(836 \pm 124 \mathrm{~ms})$. The results did not reveal any difference across conditions ( $p s>$ $0.1)$.

Regarding the Self-Construal scale, the participants were more prone to be interdependent rather than independent (5.09 \pm 0.56 vs. $4.56 \pm 0.69, t(23)=3.04, p=0.006)$. This result should be considered as a manifestation of the collectivistic cultural orientation among Chinese people.

Finally, regarding the subjective reported degree of happiness associated with gambling outcomes, a $2 \times 2 \times 2$ ANOVA involving Self-construal Type, Beneficiary, and Outcome was conducted. The participants felt happier for wins $(5.02 \pm 0.15)$ than for losses $(4.28 \pm 0.23)\left(F(1,23)=12.82, p=0.002, \eta_{p}^{2}\right.$ $=0.25$ ). Moreover, we found a significant interaction between Self-construal Type and Beneficiary $(F(1,23)=$ $\left.11.66, p=0.002, \eta_{p}^{2}=0.24\right)$. Simple effect analysis indicated that when gambling for self, there was no difference between self-construal priming conditions (independent: $4.54 \pm 1.08$, interdependent: $4.75 \pm 1.12 ; p=0.153$ ); in contrast, gambling for mother evoked a marginally significantly higher score in the independent self-construal condition $(4.88 \pm 1.23)$ than in the interdependent self-construal condition $(4.44 \pm 1.43 ; p=$ $0.065)$.

\section{ERP results}

The dFRN component For the dFRN amplitude, a repeatedmeasures ANOVA showed that the main effect of Selfconstrual Type was not significant, $F(1,23)=0.44, p=$ $0.51, \eta_{p}^{2}=0.02$, such that independent self-construal $(-2.46 \pm$ $1.50 \mu \mathrm{V})$ and interdependent self-construal $(-2.58 \pm 1.27 \mu \mathrm{V})$ evoked a comparable dFRN. The main effect of Beneficiary was not significant, $F(1,23)=1.30, p=0.27, \eta_{p}^{2}=0.05$, with gambling for self $(-2.66 \pm 1.35 \mu \mathrm{V})$ and gambling for mother $(-2.39 \pm 1.51 \mu \mathrm{V})$ evoking a comparable dFRN. The interaction between Self-construal Type and Beneficiary was significant, $F(1,23)=8.15, p=0.009, \eta_{p}^{2}=0.26$. Simple effect analysis indicated that in the Interdependent self-construal condition, gambling for self $(-2.53 \pm 1.45 \mu \mathrm{V})$ and for mother $(-2.63 \pm 1.37 \mu \mathrm{V})$ evoked a comparable dFRN $(p=0.70)$; in the Independent self-construal condition, gambling for self ($2.78 \pm 1.87 \mu \mathrm{V})$ evoked a larger dFRN than for mother $(-2.14$ $\pm 1.42 \mu \mathrm{V} ; p=0.03$, Cohen's $d=0.31$; see Fig. 2). From another perspective, when gambling for self, the dFRN showed no difference between the two self-construal conditions $(p=0.41)$; when gambling for mother, the dFRN was larger in the interdependent self-construal condition than in the independent self-construal condition $(p=0.002)$.

The P3 component For the P3 amplitude, a repeated-measure ANOVA showed that none of the main effects and the interactions were significant ( $p s>0.10)$.

\section{Discussion}

The aim of the present study was to investigate whether the response to rewards for self versus for mother would be affected by the way in which people construe the self at a given moment. The results of the self-construal scale showed that the participants were more prone to be interdependent than independent, confirming the collectivistic cultural orientation of the Chinese people (Varnum et al., 2013). In the gambling task, interdependent self-construal priming resulted in an equal FRN response to rewards for self and for mother, whereas independent priming induced a greater FRN response to rewards for self than for mother. Altogether, while previous studies have reported that gambling for self and mother evoked a comparable FRN (X. Zhu, Wang, et al., 2016; X. Zhu, Zhang, et al., 2015), the present study found that the FRN elicited by one's own rewards was larger than that elicited by mothers' rewards when the uniqueness of the self was highlighted. These findings suggest that the modulation of self-construal priming on the response to rewards for self versus for others is flexible and beneficiary-specific. Inducing the notion of the self that includes close others leads to similar response to rewards for self and for friends (Varnum et al., 2013), whereas inducing the notion of the self as autonomous and bounded leads to a stronger response to personal rewards than to mothers' rewards (this study).

Generally, watching close others receiving rewards activates the same neural network for the processing of one's own rewards, but the activation of reward circuit is weaker in the "close other" condition than the self condition (for a review, see Morelli, Sacchet, \& Zaki, 2015). As mentioned in the Introduction, Varnum et al. (2013) showed that when participants were primed with the connections with close 

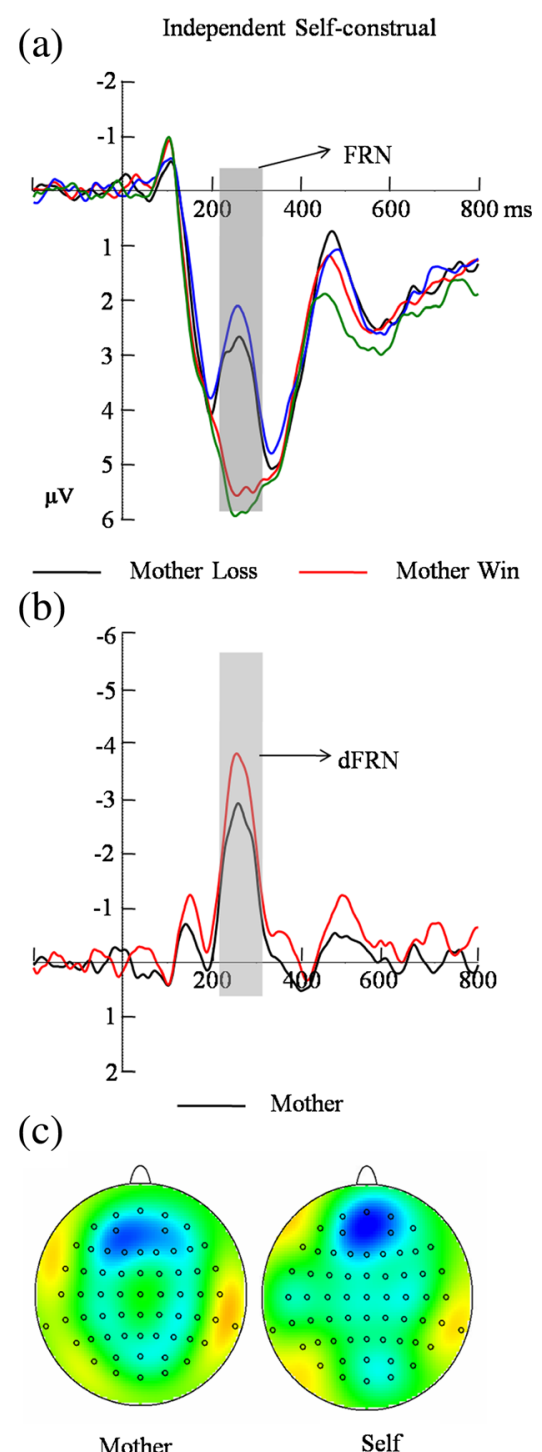

Mother

Self

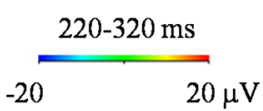

Fig. 2 Left panel: Independent self-construal conditions. Right panel: Interdependent self-construal conditions. (a) The grand-average ERP waveforms evoked by the presentation of outcome feedback (averaged across channels FC1, FCz, FC2, Cz, C1, and C2). (b) The ERP difference

others (i.e., interdependent self-construal), the difference in the ventral striatum activation between "winning for close others" and "winning for oneself" disappeared. The value of interdependence is emphasized in Chinese cultural contexts, and close others are incorporated into the self-concept (Markus \& Kitayama, 1991). Regarding that, one might expect that the neural response to rewards for mother and for self would be equal in Chinese culture whatsoever. Indeed, two of our previous ERP studies have demonstrated that Chinese people experience rewards for mother as strongly as they experience rewards for themselves (X. Zhu, Wang, et al., 2016; X. Zhu, Zhang, et al., 2015). However, the present study reveals that the ERP response to reward for mother and for self
Interdependent Self-construal
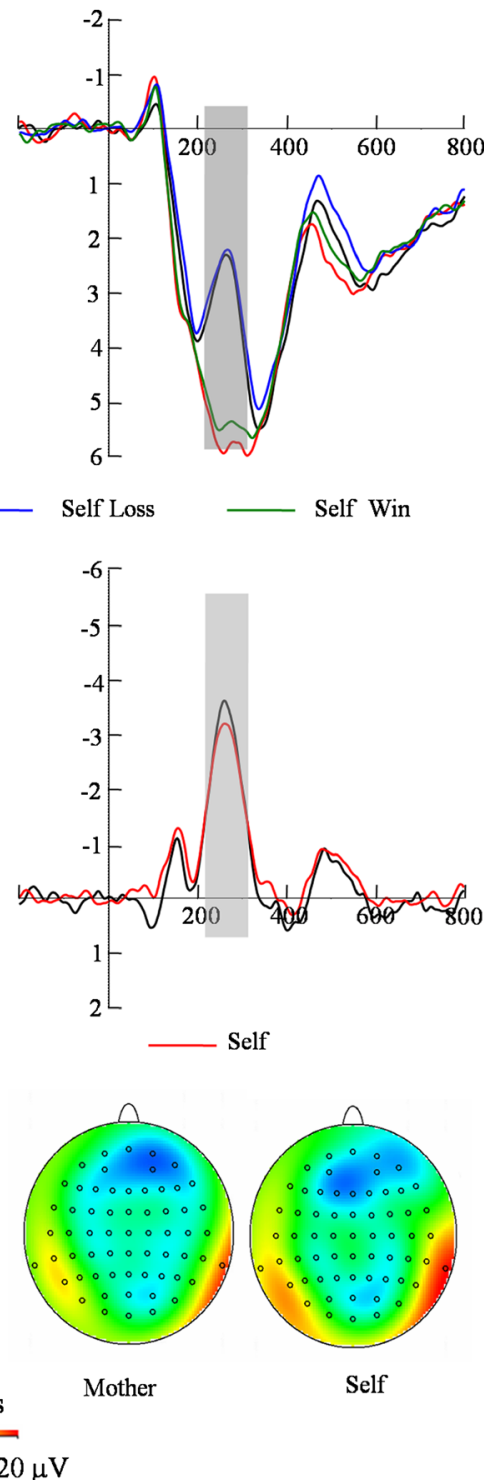

waves (also averaged across the six channels). For both (a) and (b), the gray-shaded areas indicate the time window $(220-320 \mathrm{~ms})$ in which the dFRN was measured. (c) Topographical maps of the dFRN

differentiated among Chinese participants when the uniqueness of self was emphasized.

In the present study, the patterns of the FRN results in the interdependent self-construal condition was consistent with our previous studies in which no self-construal priming was used (X. Zhu, Wang, et al., 2016; X. Zhu, Zhang, et al., 2015). This consistency across studies suggests that the default self in Chinese people is an interdependent self (Sui, Zhu, \& Chiu, 2007). However, independent priming induced a greater FRN response to rewards for self than for mother. In our opinion, the independent priming in this study has momentarily changed the dominant self-construal type from the default interdependent self to an independent self. The present results 
extend the findings of our previous studies (X. Zhu, Wang, et al., 2016; X. Zhu, Zhang, et al., 2015) that the switching towards independent self-construal is associated with changes in neural responses to rewards for mother versus self. In other words, although the neural response to rewards for mother and for self is similar in Chinese culture, this phenomenon is changeable and sensitive to the self-construal context.

What neural mechanisms have accounted for the observed modulation effect of self-construal on the FRN response to rewards for mother? Given the nature of the self-construal priming in the present study, a tentative explanation for the current results lies in the brain areas that are associated with both reward processing and self-processing. One of the most likely candidates is the $\mathrm{mPFC}$, which is not only involved in outcome evaluation but also self-processing. The response of the mPFC in self-processing is flexible and dynamic in nature (Chen, Wagner, Kelley, Powers, \& Heatherton, 2013; Chiao et al., 2009). For example, one fMRI study found that exposing to Chinese pictorial cultural icons decreased the neural difference between the mother and the self conditions, while the priming of Western culture showed a reversed effect (Ng et al., 2010). ERP localization studies have suggested that the FRN is generated around the mPFC (M. X. Cohen et al., 2011; Walsh \& Anderson, 2012). Seeing that the mPFC is involved in both self-construal and outcome feedback processing, we infer that the enhanced sensitivity to individual self enlarged the difference of mPFC activation between "gambling for mother" and "gambling for self" after independent self-construal priming.

Previous fMRI studies have indicated that different kinds of cultural primes reliably elicit an individualistic or a collectivistic cultural orientation within the same individual (Chiao et al., 2010; Ng et al., 2010). However, it remains unclear whether individualistic or collectivistic cultural primes (e.g., pictorial cultural icons) would influence the neural response to rewards for mother in the same way as self-construal priming in this study. If the individualistic cultural primes can also differentiate the neural response to rewards for mother and for self, then one could conclude that reward processing is influenced not only by individual self-representation, but also the broader cultural values.

One limitation is that the present study did not examine the individual difference in self-construal. Chiao et al. (2009) found that self-processing within the MPFC varies as a function of self-construal type: the participants were divided into "individualists" and "collectivists" groups based on the selfconstrual scale score; individualists showed greater mPFC activation to general self-descriptions, whereas collectivists showed greater mPFC activation to contextual selfdescriptions (i.e., judging their traits relative to their mothers). It remains unclear whether the self-construal priming effect on the rewards for mother varies as a function of self-construal type. Additionally, we only included Chinese people in the present study. For Chinese people, interdependence is the default self-construal orientation; in contrast, independence is the dominant self-construal orientation in Western culture. In our opinion, the current findings have general implications about the role of self-construal in the processing of outcome feedback for self and for others. This study, however, has only investigated a specific interdependent culture (Chinese) and a specific other (mother). Due to limitations in resources, we currently are unable to examine whether the same results could also be found in Western culture and for non-specific others in a full-factor design. Therefore, the generalizability of our conclusions remains to be clarified by future studies.

Another potential problem is that we asked participants to finish the Self-Construal scale after the formal task, because we were afraid that this scale per se might induce the priming effect, and therefore would become a confounding factor. However, we could not rule out the possibility that the standard self-construal priming has affected the self-report scores of the Self-Construal scale. Follow-up studies may address this procedure issue with alterative task designs. Finally, the limited sample size might have prevented us from discovering more interesting findings, such as the role of individual difference in self-construal. Future research with a larger sample size would be helpful to revisit the negative results in the current study.

Finally, the results of self-report happiness rating of outcome feedback were surprising, since the happiness level associated with outcome feedback of gambling for mother showed a tendency to be higher in the independent selfconstrual condition than in the interdependent self-construal condition. However, this tendency failed to reach significance, and follow-up studies might be needed to examine its reliability. If this tendency really existed, one might question why it showed different pattern with the FRN results. Our explanation is that the FRN reflects the bottom-up, automatic stage of outcome evaluation (Yang, Tang, Gu, Luo, \& Luo, 2015), and therefore its pattern might deviate from conscious self-reports.

To sum up, the present ERP study indicated that emphasizing the independence of the self enhances the distinction between self and mother during outcome evaluation, while emphasizing the interdependence of the self diminishes this distinction. Combining the results of two of our previous studies (X. Zhu, Wang, et al., 2016; X. Zhu, Zhang, et al., 2015), we suggest that the neural responses to reward for mother and for self are dynamic by nature.

Acknowledgements This research was supported by the National Natural Science Foundation of China (31300846, 31571124, 31600931).

\section{References}

Amodio, D. M., Bartholow, B. D., \& Ito, T. A. (2014). Tracking the dynamics of the social brain: ERP approaches for social cognitive and affective neuroscience. Social Cognitive \& Affective Neuroscience, 9(3), 385-393. doi: https://doi.org/10.1093/scan/ nst177 
Bress, J. N., \& Hajcak, G. (2013). Self-report and behavioral measures of reward sensitivity predict the feedback negativity. Psychophysiology, 50(7), 610-616. doi: https://doi.org/10.1111/ psyp. 12053

Chen, P. H. A., Wagner, D. D., Kelley, W. M., Powers, K. E., \& Heatherton, T. F. (2013). Medial prefrontal cortex differentiates self from mother in Chinese: Evidence from self-motivated immigrants. Culture and Brain, 1(1), 3-15. doi: https://doi.org/10.1007/s40167013-0001-5

Chiao, J. Y., Harada, T., Komeda, H., Li, Z., Mano, Y., Saito, D.,... Iidaka, T. (2009). Neural basis of individualistic and collectivistic views of self. Human Brain Mapping, 30(9), 2813-2820. doi: https://doi.org/ 10.1002/hbm.20707

Chiao, J. Y., Harada, T., Komeda, H., Li, Z., Mano, Y., Saito, D.,... Iidaka, T. (2010). Dynamic cultural influences on neural representations of the self. Journal of Cognitive Neuroscience, 22(1), 1-11. doi: https:// doi.org/10.1162/jocn.2009.21192

Cohen, J. (1973). Eta-squared and partial eta-squared in fixed factor ANOVA designs. Educational and Psychological Measurement, 33(1), 107-112. doi: https://doi.org/10.1177/001316447303300111

Cohen, M. X., Wilmes, K., \& van de Vijver, I. (2011). Cortical electrophysiological network dynamics of feedback learning. Trends in Cognitive Sciences, 15(12), 558-566. doi: https://doi.org/10.1016/j. tics.2011.10.004

Colzato, L. S., de Bruijn, E. R., \& Hommel, B. (2012). Up to "me" or up to "us"? The impact of self-construal priming on cognitive self-other integration. Frontiers in Psychology, 3, 341. doi: https://doi.org/10. 3389/fpsyg.2012.00341

Cross, S. E., Hardin, E. E., \& Gercek-Swing, B. (2011). The what, how, why, and where of self-construal. Personality and Social Psychology Review, 15(2), 142-179. doi: https://doi.org/10.1177/ 1088868310373752

Faul, F., Erdfelder, E., Lang, A. G., \& Buchner, A. (2007). G*Power 3: A flexible statistical power analysis program for the social, behavioral, and biomedical sciences. Behavior Research Methods, 39(2), 175191. doi: https://doi.org/10.3758/BF03193146

Foti, D., Weinberg, A., Bernat, E. M., \& Proudfit, G. H. (2015). Anterior cingulated activity to monetary loss and basal ganglia activity to monetary gain uniquely. Clinical Neurophysiology, 126(7), 13381347. doi: https://doi.org/10.1016/j.clinph.2014.08.025

Gardner, W. L., Gabriel, S., \& Lee, A. Y. (1999). "I" value freedom, but "we" value relationships: Self-construal priming mirrors cultural differences in judgment. Psychological Science, 10(4), 321-326. doi: https://doi.org/10.1111/1467-9280.00162

Gehring, W. J., \& Willoughby, A. R. (2002). The medial frontal cortex and the rapid processing of monetary gains and losses. Science, 295(5563), 2279-2282. doi: https://doi.org/10.1126/science. 1066893

Greenhouse, S. W., \& Geisser, S. (1959). On methods in the analysis of profile data. Psychometrika, 24(2), 95-112. doi: https://doi.org/10. 1007/BF02289823

Gu, R., Feng, X., Broster, L. S., Yuan, L., Xu, P., \& Luo, Y.j. (2017). Valence and magnitude ambiguity in feedback processing. Brain and Behavior, 7(7), e00672. doi: https://doi.org/10.1002/brb3.672

Gu, R., Ge, Y., Jiang, Y., \& Luo, Y. J. (2010). Anxiety and outcome evaluation: The good, the bad and the ambiguous. Biological Psychology, 85(2), 200-206. doi: https://doi.org/10.1016/j. biopsycho.2010.07.001

Gu, R., Huang, Y. X., \& Luo, Y. J. (2010). Anxiety and feedback negativity. Psychophysiology, 47(5), 961-967. doi: https://doi.org/10. 1111/j.1469-8986.2010.00997.x

Gu, R., Wu, T., Jiang, Y., \& Luo, Y. J. (2011). Woulda, coulda, shoulda: The evaluation and the impact of the alternative outcome. Psychophysiology, 48(10), 1354-1360. doi: https://doi.org/10.1111/ j.1469-8986.2011.01215.x
Hajcak, G., Dunning, J. P., \& Foti, D. (2009). Motivated and controlled attention to emotion: Time-course of the late positive potential. Clinical Neurophysiology, 120(3), 505-510. doi: https://doi.org/10. 1016/j.clinph.2008.11.028

Hajcak, G., Moser, J. S., Holroyd, C. B., \& Simons, R. F. (2007). It's worse than you thought: The feedback negativity and violations of reward prediction in gambling tasks. Psychophysiology, 44(6), 905912. doi: https://doi.org/10.1111/j.1469-8986.2007.00567.x

Han, S., \& Humphreys, G. (2016). Self-construal: A cultural framework for brain function. Current Opinion in Psychology, 8, 10-14. doi: https://doi.org/10.1016/j.copsyc.2015.09.013

Heatherton, T. F., Wyland, C. L., Macrae, C. N., Demos, K. E., Denny, B. T., \& Kelley, W. M. (2006). Medial prefrontal activity differentiates self from close others. Social Cognitive \& Affective Neuroscience, 1(1), 18-25. doi: https://doi.org/10.1093/scan/nsl001

Holroyd, C. B., \& Krigolson, O. E. (2007). Reward prediction error signals associated with a modified time estimation task. Psychophysiology, 44(6), 913-917. doi: https://doi.org/10.1111/j. 1469-8986.2007.00561.x

Holroyd, C. B., Pakzad-Vaezi, K. L., \& Krigolson, O. E. (2008). The feedback correct-related positivity: Sensitivity of the event-related brain potential to unexpected positive feedback. Psychophysiology, 45(5), 688-697. doi: https://doi.org/10.1111/j.1469-8986.2008. 00668.x

Jiang, C., Varnum, M. E., Hou, Y., \& Han, S. (2014). Distinct effects of self-construal priming on empathic neural responses in Chinese and Westerners. Social Neuroscience, 9(2), 130-138. doi: https://doi.org/ 10.1080/17470919.2013.867899

Krishna, A., Zhou, R., \& Zhang, S. (2008). The effect of self-construal on spatial judgments. Journal of Consumer Research, 35(2), 337-348. doi: https://doi.org/10.1086/588686

Lange, S., Leue, A., \& Beauducel, A. (2012). Behavioral approach and reward processing: Results on feedback-related negativity and P3 component. Biological Psychology, 89(2), 416-425. doi: https://doi. org/10.1016/j.biopsycho.2011.12.004

Leng, Y., \& Zhou, X. L. (2010). Modulation of the brain activity in outcome evaluation by interpersonal relationship: An ERP study. Neuropsychologia, 48(2), 448-455.

Lockwood, P. L., Apps, M. A., Valton, V., Viding, E., \& Roiser, J. P. (2016). Neurocomputational mechanisms of prosocial learning and links to empathy. Proceedings of the National Academy of Sciences of the United States of America, 113(35), 9763-9768. doi: https:// doi.org/10.1073/pnas.1603198113

Macrae, C. N., Moran, J. M., Heatherton, T. F., Banfield, J. F., \& Kelley, W. M. (2004). Medial prefrontal activity predicts memory for self. Cerebral Cortex, 14(6), 647-654. doi: https://doi.org/10.1093/ cercor/bhh025

Markus, H. R., \& Kitayama, S. (1991). Culture and the self: Implications for cognition, emotion, and motivation. Psychological Review, 98(2), 224-253. doi: https://doi.org/10.1037/0033-295X.98.2.224

Miltner, W. H. R., Braun, C. H., \& Coles, M. G. H. (1997). Event-related brain potentials following incorrect feedback in a time-estimation task: Evidence for a "generic" neural system for error detection. Journal of Cognitive Neuroscience, 9(6), 788-798. doi: https://doi. org/10.1162/jocn.1997.9.6.788

Mobbs, D., Yu, R., Meyer, M., Passamonti, L., Seymour, B., Calder, A. J., ... Dalgleish, T. (2009). A key role for similarity in vicarious reward. Science, 324(5929), 900. doi: https://doi.org/10.1126/science. 1170539

Morelli, S. A., Sacchet, M. D., \& Zaki, J. (2015). Common and distinct neural correlates of personal and vicarious reward: A quantitative meta-analysis. Neuroimage, 112, 244-253. doi: https://doi.org/10. 1016/j.neuroimage.2014.12.056

Ng, S. H., Han, S., Mao, L., \& Lai, J. C. (2010). Dynamic bicultural brains: fMRI study of their flexible neural representation of self and significant others in response to culture primes. Asian Journal 
of Social Psychology, 13(2), 83-91. doi: https://doi.org/10.1111/j. 1467-839X.2010.01303.x

Nieuwenhuis, S., Heslenfeld, D. J., von Geusau, N. J. A., Mars, R. B., Holroyd, C. B., \& Yeung, N. (2005). Activity in human rewardsensitive brain areas is strongly context dependent. Neuroimage, 25(4), 1302-1309. doi: https://doi.org/10.1016/j.neuroimage.2004. 12.043

Obhi, S. S., Hogeveen, J., \& Pascual-Leone, A. (2011). Resonating with others: The effects of self-construal type on motor cortical output. Journal of Neuroscience, 31(41), 14531-14535. doi: https://doi.org/ 10.1523/JNEUROSCI.3186-11.2011

Polezzi, D., Sartori, G., Rumiati, R., Vidotto, G., \& Daum, I. (2010). Brain correlates of risky decision-making. Neuroimage, 49(2), 1886-1894. doi: https://doi.org/10.1016/j.neuroimage.2009.08.068

Proudfit, G. H. (2015). The reward positivity: From basic research on reward to a biomarker for depression. Psychophysiology, 52(4), 449-459. doi: https://doi.org/10.1111/psyp. 12370

Ray, R. D., Shelton, A. L., Hollon, N. G., Matsumoto, D., Frankel, C. B., Gross, J. J., \& Gabrieli, J. D. (2010). Interdependent self-construal and neural representations of self and mother. Social Cognitive \& Affective Neuroscience, 5(2-3), 318-323. doi: https://doi.org/10. 1093/scan/nsp039

Sambrook, T. D., \& Goslin, J. (2015). A neural reward prediction error revealed by a meta-analysis of ERPs using great grand averages. Psychological Bulletin, 141(1), 213-235. doi: https://doi.org/10. 1037/bul0000006

San Martín, R. (2012). Event-related potential studies of outcome processing and feedback-guided learning. Frontiers in Human Neuroscience, 6, 304. doi: https://doi.org/10.3389/fnhum.2012. 00304

Singelis, T. M. (1994). The measurement of independent and interdependent self-construals. Personality and Social Psychology Bulletin, 20(5), 580-591. doi: https://doi.org/10.1177/0146167294205014

Sui, J., \& Han, S. (2007). Self-construal priming modulates neural substrates of self-awareness. Psychological Science, 18(10), 861-866. doi: https://doi.org/10.1111/j.1467-9280.2007.01992.x

Sui, J., Hong, Y. Y., Hong Liu, C., Humphreys, G. W., \& Han, S. (2013). Dynamic cultural modulation of neural responses to one's own and friend's faces. Social Cognitive \& Affective Neuroscience, 8(3), 326332. doi: https://doi.org/10.1093/scan/nss001

Sui, J., Zhu, Y., \& Chiu, C. Y. (2007). Bicultural mind, self-construal, and self-and mother-reference effects: Consequences of cultural priming on recognition memory. Journal of Experimental Social Psychology, 43(5), 818-824. doi: https://doi.org/10.1016/j.jesp.2006.08.005

Van Baaren, R. B., Maddux, W. W., Chartrand, T. L., De Bouter, C., \& van Knippenberg, A. (2003). It takes two to mimic: Behavioral consequences of self-construals. Journal of Personality and Social Psychology, 84(5), 1093-1102. doi: https://doi.org/10.1037/00223514.84.5.1093

van Prooijen, J. W., \& Zwenk, F. (2009). Self-construal level and voice procedures: The individual self as psychological basis for procedural fairness effects. Journal of Experimental Social Psychology, 45(2), 392-397. doi: https://doi.org/10.1016/j.jesp.2008.10.008

Varnum, M. E., Shi, Z., Chen, A., Qiu, J., \& Han, S. (2013). When "Your" reward is the same as "My" reward: Self-construal priming shifts neural responses to own vs. friends' rewards. Neuroimage, 87C, 164-169. doi: https://doi.org/10.1016/j.neuroimage.2013.10. 042
Vazire, S. (2016). Editorial. Social Psychological and Personality Science, 7(1), 3-7. doi: https://doi.org/10.1177/1948550615603955

Walsh, M. M., \& Anderson, J. R. (2012). Learning from experience: Event-related potential correlates of reward processing, neural adaptation, and behavioral choice. Neuroscience \& Biobehavioral Reviews, 36(8), 1870-1884. doi: https://doi.org/10.1016/j. neubiorev.2012.05.008

Wang, C., Ma, Y., \& Han, S. (2014). Self-construal priming modulates pain perception: Event-related potential evidence. Cognitive Neuroscience, 5(1), 3-9. doi: https://doi.org/10.1080/17588928. 2013.797388

Wang, G., Mao, L., Ma, Y., Yang, X., Cao, J., Liu, X.,... Han, S. (2012). Neural representations of close others in collectivistic brains. Social Cognitive \& Affective Neuroscience, 7(2), 222-229. doi: https://doi. org $/ 10.1093 / \mathrm{scan} / \mathrm{nsr} 002$

Wang, Y., Yuan, Q., \& Xu, Q. (2008). A preliminary study on selfconstructionals scale of Chinese-version. Chinese Journal of Clinical Psychology, 16(6), 602-604.

Wu, Y., \& Zhou, X. L. (2009). The P300 and reward valence, magnitude, and expectancy in outcome evaluation. Brain Research, 1286, 114122. doi: https://doi.org/10.1016/j.brainres.2009.06.032

Wuyun, G., Shu, M., Cao, Z., Huang, W., Zou, X., Li, S.,... Wu, Y. (2014). Neural representations of the self and the mother for Chinese individuals. Plos One, 9(3), e91556. doi: https://doi.org/ 10.1371/journal.pone.0091556

Yang, Q., Tang, P., Gu, R., Luo, W., \& Luo, Y. J. (2015). Implicit emotion regulation affects outcome evaluation. Social Cognitive and Affective Neuroscience, 10(6), 824-831. doi: https://doi.org/10. $1093 /$ scan/nsu124

Yeung, N., \& Sanfey, A. G. (2004). Independent coding of reward magnitude and valence in the human brain. Journal of Neuroscience, 24(28), 6258-6264. doi: https://doi.org/10.1523/JNEUROSCI. 4537-03.2004

Zendel, B. R., \& Alain, C. (2014). Enhanced attention-dependent activity in the auditory cortex of older musicians. Neurobiol Aging, 35(1), 55-63. doi: https://doi.org/10.1016/j.neurobiolaging.2013.06.022

Zhu, X., Gu, R., Wu, H., \& Luo, Y. (2015). Self-reflection modulates the outcome evaluation process: Evidence from an ERP study. International Journal of Psychophysiology, $98(3$ Pt 1), 389-393. doi: https://doi.org/10.1016/j.ijpsycho.2015.08.001

Zhu, X., Wang, L., Yang, S., Gu, R., Wu, H., \& Luo, Y. J. (2016). The motivational hierarchy between the personal self and close others in the Chinese brain: An ERP study. Frontiers in Psychology, 7, 1467. doi: https://doi.org/10.3389/fpsyg.2016.01467

Zhu, X., Wu, H., Yang, S., \& Gu, R. (2016). Motivational hierarchy in the Chinese brain: Primacy of the individual self, relational self, or collective self? Frontiers in Psychology, 7, 877. doi: https://doi.org/10. 3389/fpsyg.2016.00877

Zhu, X., Wu, H., Yang, S., \& Gu, R. (2017). The influence of selfconstrual type on outcome evaluation: Evidence from eventrelated potentials. International Journal of Psychophysiology, 112, 64-69. doi: https://doi.org/10.1016/j.ijpsycho.2016.12.010

Zhu, X., Zhang, Y., Yang, S., Wu, H., Wang, L., \& Gu, R. (2015). The motivational hierarchy between self and mother: Evidence from the feedback-related negativity. Acta Psychologica Sinica, 47(6), 807813. doi: https://doi.org/10.3724/SP.J.1041.2015.00807

Zhu, Y., Zhang, L., Fan, J., \& Han, S. (2007). Neural basis of cultural influence on self-representation. Neuroimage, 34(3), 1310-1316. doi: https://doi.org/10.1016/j.neuroimage.2006.08.047 